\title{
Dark energy and extending the geodesic equations of motion: connecting the galactic and cosmological length scales
}

\section{A. D. Speliotopoulos}

Received: 23 May 2010 / Accepted: 16 June 2010 / Published online: 4 July 2010

(C) The Author(s) 2010. This article is published with open access at Springerlink.com

\begin{abstract}
Recently, an extension of the geodesic equations of motion using the Dark Energy length scale was proposed. Here, we apply this extension to analyzing the motion of test particles at the galactic scale and longer. A cosmological check of the extension is made using the observed rotational velocity curves and core sizes of 1,393 spiral galaxies. We derive the density profile of a model galaxy using this extension, and with it, we calculate $\sigma_{8}$ to be $0.73_{ \pm 0.12}$; this is within experimental error of the WMAP value of $0.761_{-0.048}^{+0.049}$. We then calculate $R_{200}$ to be $206_{ \pm 53} \mathrm{kpc}$, which is in reasonable agreement with observations.
\end{abstract}

Keywords Dark energy · Galactic density profile · Density fluctuations · Extensions of the geodesic equations of motion - Galactic rotation curves

\section{Introduction}

In a previous paper [1], we constructed an extension of the geodesic equations of motion (GEOM). This construction is possible because with the discovery of Dark Energy, $\Lambda_{\mathrm{DE}}=\left(7.21_{-0.84}^{+0.82}\right) \times 10^{-30} \mathrm{~g} / \mathrm{cm}^{3}$ [2-4], there is now a length scale, $\lambda_{\mathrm{DE}}=$ $c /\left(\Lambda_{\mathrm{DE}} G\right)^{1 / 2}$, associated with the universe. As this length scale is also not associated with the mass of any known particle, this extension does not violate various statements of the equivalence principle. Importantly, the extension does not change the GEOM

\footnotetext{
A. D. Speliotopoulos $(\varangle)$

Department of Physics, University of California, Berkley, CA 94720, USA

e-mail: ads@berkeley.edu
}

\section{A. D. Speliotopoulos}

Department of Mathematics, Golden Gate University,

San Francisco, CA 94105, USA 
for massless particles, and thus astronomical observations of the universe-which are based on the trajectory of photons - remain unchanged. At $14,010_{820}^{800} \mathrm{Mpc}$, the shear scale of $\lambda_{\mathrm{DE}}$ ensures that effects of this extension will not have already been observed either in the motion of bodies in the solar system, or in terrestrial experiments. Indeed, by analyzing the effects of the extension at these length scales, we established a lower bound to $\alpha_{\Lambda}$. This $\alpha_{\Lambda}$ is the only free parameter in the theory, and gives the power law dependence of the extension on the ratio $c^{2} R / \Lambda_{\mathrm{DE}} G$, where $R$ is the Ricci scalar.

At the conclusion of [1], we argued that it is only at galactic length scales and longer will we expect effects of the extension to be relevant. A study of these effects is the focus of this paper. In particular, we analyze the restrictions on our extension of the GEOM due to both observations of galactic structure, and recent measurements by WMAP. We find that these observations and measurements do not rule out our extension of the GEOM. To the contrary, by applying the extension to an analysis of the motion of stars in spiral galaxies, we are able to calculate $\sigma_{8}$ - the rms fluctuation in the density of matter at $8 h^{-1} \mathrm{Mpc}$ - to be $0.73_{ \pm 0.12}$. This value is in excellent agreement with the WMAP measurement of $0.761_{-0.048}^{+0.049}$ for $\sigma_{8}$. We are also able to calculate $R_{200}$ - the distance from the center of a galaxy at which the density of matter equals 200 times that of the critical density - to be $206_{ \pm 53} \mathrm{kpc}$, which is in reasonable agreement with observations. Calculations of both quantities are possible because of an unexpected connection between the effects of our extension on the motion of test particles at galactic scales with cosmological length scales. This connection allows us to set a definite value of $1.56_{ \pm 0.10}$ for $\alpha_{\Lambda}$ that is equal to the rough lower bound on $\alpha_{\Lambda}$ established in [1] for effects of the extension to be unobservable at solar system and terrestrial scales.

This stringent test of the extended GEOM is only possible because of the scale of $\lambda_{\mathrm{DE}}$. With a value of $14,010_{820}^{800} \mathrm{Mpc}$, it is expected that any effects of the extension will only be apparent at the galactic scale or longer. (Indeed, $\lambda_{\mathrm{DE}}$ is so large that it is only because of the nonlinear dependence of the extension on $R$ that effects at the galactic scale are apparent at all.) It is also precisely at the galactic length scale where deviations from motion under Newtonian gravity appear. Using a simple model of a spiral galaxy, we are able to determine the density profile of the galaxy by applying our extension of the GEOM to the motion of stars within it. We find that effects of our extension necessarily extend beyond the galactic scale. Not unexpectedly, at these scales the $1 / r$ interaction potential that is expected between galaxies from Newtonian gravity is now logarithmic, which is consistent with the interaction potential between galaxies and galactic clusters inferred through observations. At even larger length scales, we find that the predicted density profile for the model galaxy goes to zero exponentially fast at distances beyond the Hubble length scale, $\lambda_{H}=c / H$, (where $H=h H_{0}$, and $H_{0}=100 \mathrm{~km} / \mathrm{s} / \mathrm{Mpc}$ ) from the center of the model galaxy. While this result is certainly physically reasonable, it is surprising that such a length scale naturally appears in the theory, even though a cosmological model is not mentioned either in its construction, or in its analysis. This unexpected connection with cosmology allows us to set the value of $\alpha_{\Lambda}$ to be $1.56_{ \pm 0.10}$ using the WMAP measurements of $h$ and $\Lambda_{\mathrm{DE}}$.

We have also used this density profile to calculate explicitly $\sigma_{8}$. This calculation is possible because of four data sets in the literature, [5-13]. These data sets are the 
result of observations - made over a 30-year span-of galactic rotation curves that give both the asymptotic rotational velocity and the core sizes of 1,393 spiral galaxies. While only a very small fraction of the observed galaxies in the universe, the size of the data set and the fact that the vast majority of these observations were unbiased, allows us to obtain average values for the parameters used to characterize the model galaxy. These parameters, along with the value of $\alpha_{\Lambda}$, is used to calculate a $\sigma_{8}$ that is in excellent agreement with WMAP measurements. We are also able to use these parameters to predict a value of $206_{ \pm 53} \mathrm{kpc}$ for $R_{200}$, which is in reasonable agreement with observations.

Like Peebles' model for structure formation [14], the total density of matter for our model galaxy can be written as a sum of a asymptotic, background density, $\rho_{\text {asymp }}(\mathbf{x})$, and a linear perturbation, $\rho_{\mathrm{II}}^{1}(\mathbf{x})$. The matter that makes up $\rho_{\text {asymp }}(\mathbf{x})$ does not contribute to the motion of stars within the galaxy, while the matter that makes up $\rho_{\mathrm{II}}^{1}(\mathbf{x})$ does. Unlike Peebles' model, however, $\rho_{\text {asymp }}(\mathbf{x})$ is not a constant, but instead varies inversely with distance from the galactic core, and dies off exponentially fast beyond the Hubble scale. Moreover, the form of $\rho_{\text {asymp }}(\mathbf{x})$ depends only on the dimensionality of spacetime and $\alpha_{\Lambda}$, while the scale at which it decreases depends only on $\lambda_{\mathrm{DE}}$. In this sense, $\rho_{\text {asymp }}(\mathbf{x})$ is universal, and does not depend on the detail structure of the galaxy. This is in contrast to $\rho_{\mathrm{II}}^{1}(\mathbf{x})$, which depends explicitly on the structure of the galaxy near the galactic core both in form and in scale.

While $\rho_{\text {asymp }}(\mathbf{x})$ does not contribute to the motion of stars within galaxies, it does contribute to the deflection of light. By construction, the extended GEOM does not affect the equations of motion for massless particles. Light still travels along geodesics, and the degree of the deflection of light is determined by the total local density of matter. As such, using the deflection of light to measure the local matter density will result in a measurement of $\rho_{\text {asymp }}(\mathbf{x})+\rho_{\mathrm{II}}^{1}(\mathbf{x})$. In contrast, the motion of stars in galaxies are affected by our extension of the GEOM. As such, their motion is determined solely by $\rho_{\mathrm{II}}^{1}(\mathbf{x})$, and thus when this motion is used to determine the local matter density, what is measured is only the matter that makes up $\rho_{\mathrm{II}}^{1}(\mathbf{x})$. As we find $\rho_{\mathrm{II}}^{1}<<\rho_{\text {asymp }}$ outside of a few galactic core radii, the presence of the vast majority of matter in the universe can only be inferred by though the effects that the local density of matter has on the trajectory of light.

The rest of the paper is divided into five parts. In the first part, an overview of the extended GEOM is given, and the properties of the extension needed in this paper is outlined. In the second part, we introduce our model of the galaxy, and using the extended GEOM, we derive the density profile of the galaxy given a rotational velocity curve for it. We show that this density dies off exponentially fast beyond a fixed distance from the galaxy. In the third part, we use WMAP measurements of $h$ to determine $\alpha_{\Lambda}$, and calculate $\sigma_{8}$ and $R_{200}$ using the density profile of our model galaxy and observational data on galactic rotation curves from the literature. The value for $\sigma_{8}$ is then compared with WMAP measurements. In the fourth part, we calculate the gravitational potential for the model galaxy, and determine which portion of the density can be determined through direct observations of the motion of stars in the galaxy, and which can only be determined through the deflection of light. Concluding remarks can then be found in the last part. 


\section{An overview of the extended GEOM}

While there is currently no consensus as to the nature of Dark Energy, modifications to Einstein's equations to include the cosmological constant are both well known and minimal. In addition, WMAP measured the ratio of the pressure to energy density ratio for Dark Energy to be $-0.967_{-0.072}^{+0.073}$; this is within experimental error of -1 , the ratio expected for the cosmological constant. We thus identify Dark Energy with the cosmological constant in this paper, and require only that $\Lambda_{\mathrm{DE}}$ changes so slowly that it can be considered a constant in our analysis. Einstein's field equations are then

$$
R_{\mu \nu}-\frac{1}{2} g_{\mu \nu} R+\frac{\Lambda_{\mathrm{DE}} G}{c^{2}} g_{\mu \nu}=-\frac{8 \pi G}{c^{4}} T_{\mu \nu}
$$

where $T_{\mu \nu}$ is the energy-momentum tensor for matter, $R_{\mu \nu}$ is the Ricci tensor, Greek indices run from 0 to 3 , and the signature of $g_{\mu \nu}$ is $(1,-1,-1,-1)$.

The extended GEOM for a test particle with mass, $m$, is obtained from the Lagrangian

$$
\mathcal{L}_{\mathrm{Ext}} \equiv \mathfrak{R}\left[c^{2} R / \Lambda_{\mathrm{DE}} G\right]\left(g_{\mu \nu} \dot{x}^{\mu} \dot{x}^{\nu}\right)^{\frac{1}{2}}
$$

While the function $\mathfrak{R}$ is arbitrary, in [1] we argued that the simplest choice is $\mathfrak{R}(x)=$ $[1+\mathfrak{D}(x)]^{1 / 2}$, where

$$
\mathfrak{D}(x)=\chi\left(\alpha_{\Lambda}\right) \int_{x}^{\infty} \frac{d s}{1+s^{1+\alpha_{\Lambda}}}
$$

while

$$
\frac{1}{\chi\left(\alpha_{\Lambda}\right)} \equiv \int_{0}^{\infty} \frac{d s}{1+s^{1+\alpha_{\Lambda}}}=\frac{\sin \left[\pi /\left(1+\alpha_{\Lambda}\right)\right]}{\pi /\left(1+\alpha_{\Lambda}\right)}
$$

is defined so that $D(0)=1$. Here, $\alpha_{\Lambda}$ is a constant, and is the only free parameter in the theory. In [1], we showed that for the effects of the extension not to have already been observed in terrestrial experiments, $\alpha_{\Lambda}$ must be between 1.28 (for $\Lambda_{\mathrm{DE}}=$ $10^{-32} \mathrm{~g} / \mathrm{cm}^{3}$ ) and 1.58 (for $\Lambda_{\mathrm{DE}}=10^{-29} \mathrm{~g} / \mathrm{cm}^{3}$ ).

While the equations of motion derived from $\mathcal{L}_{\text {EXT }}$ is

$$
\frac{D^{2} x^{\mu}}{\partial t^{2}}=c^{2}\left(g^{\mu \nu}-\frac{v^{\mu} v^{\nu}}{c^{2}}\right) \nabla_{\nu} \log \Re\left[c^{2} R / \Lambda_{\mathrm{DE}} G\right],
$$

we are interested in the motion of stars in galaxies. Moreover, WMAP and the Supernova Legacy Survey put $\Omega_{K}=-0.011_{ \pm 0.012}$, and thus the universe is essentially flat. Indeed, WMAP's value for $h$ is determined with this assumption. As such, we are working in the nonrelativistic and weak gravity limits, and therefore take the 
metric to be $g_{\mu \nu}=\eta_{\mu \nu}+h_{\mu \nu}$. Here, $\eta_{\mu \nu}$ is the Minkowski metric, and $h_{\mu \nu}$ is a small perturbation. The only nonzero component of $h_{\mu \nu}$ is $h_{00}=2 \Phi / c^{2}$, where $\Phi$ is the Newtonian gravitational potential. Equation (1) then reduces to

$$
\nabla^{2} \Phi+2 \frac{\Lambda_{\mathrm{DE}} G}{c^{2}} \Phi=4 \pi \rho G-\Lambda_{\mathrm{DE}} G
$$

with the additional terms due to the cosmological constant.

As we are dealing with the motion of stars in galaxies, taking $\eta_{\mu \nu}$ to be the background metric would seem to be straightforward. There is one subtlety, however. Even when $T_{\mu \nu}=0$ in Eq. (1), $\Lambda_{\mathrm{DE}}$ is present, and the spacetime is not flat; at scales comparable to $\lambda_{\mathrm{DE}}$, the spacetime will be significantly different from Minkowski space even in the absence of matter. At $14,010_{820}^{800} \mathrm{Mpc}, \lambda_{\mathrm{DE}}$ is over three times the Hubble scale, $\lambda_{H}$, however, and taking the background metric to be flat is a good approximation throughout most of the physically relevant length scales. By restricting ourselves to length scales much less than $\lambda_{\mathrm{DE}}$, taking $\eta_{\mu \nu}$ as the background metric is a good approximation, and the terms proportional to $\Lambda_{\mathrm{DE}}$ in Eq. (6) can be neglected.

Using Eq. (6), Eq. (5) reduces to

$$
\frac{d^{2} \mathbf{x}}{d t^{2}} \approx-\nabla \Phi+\left(\frac{4 \pi c^{2} \chi}{\Lambda_{\mathrm{DE}}}\right)\left\{1+\left(4+\frac{8 \pi \rho}{\Lambda_{\mathrm{DE}}}\right)^{1+\alpha_{\Lambda}}\right\}^{-1} \nabla \rho
$$

in the nonrelativistic and weak gravity limits. Here, we have related $R$ to the trace of the energy-momentum tensor, $T$, using Eq. (1). We have also assumed that the spacetime is spatially symmetric, and that the particle moves through an ambient, nonrelativistic fluid with density, $\rho$. We are dealing with only gravitational forces, and thus do not differentiate between baryonic matter and Dark Matter in $\rho$. As shown in [1], the energy-momentum tensor for the fluid can be approximated as $T_{\mu \nu} \approx \rho u_{\mu} u_{\nu}$ in the nonrelativistic and weak gravity limits even though elements in the fluid propagate under the extended GEOM instead of the GEOM. Because $\Lambda_{\mathrm{DE}} \sim 10^{-30} \mathrm{~g} / \mathrm{cm}^{3}$, we expect that $8 \pi \rho / \Lambda_{\mathrm{DE}} \geq 0$ in galaxies, and thus have also used the expansion

$$
\mathfrak{D}\left(4+8 \pi \rho / \Lambda_{\mathrm{DE}}\right)=\chi \sum_{n=0}^{\infty} \frac{(-1)^{n}}{n\left(1+\alpha_{\Lambda}\right)+\alpha_{\Lambda}}\left(4+\frac{8 \pi \rho}{\Lambda_{\mathrm{DE}}}\right)^{-n\left(1+\alpha_{\Lambda}\right)-\alpha_{\Lambda}},
$$

in obtaining Eq. (7).

\section{Effects on the galactic scale}

While definitive, a first principle calculation of the galactic rotation curves using the extended GEOM to describe the motion each star in a galaxy is analytically intractable. Instead, given a rotation curve for a model galaxy, we will use the extended GEOM to derive the density profile for the galaxy, and through this profile, describe the motion of test particles within it. Not surprisingly, we find that inside the galactic core stars 
move as though they are in a Newtonian potential. Outside of the core, on the other hand, stars move as though they are in a non-Newtonian, logarithmic potential.

Since the lower bound on $\alpha_{\Lambda}$ is between 1.28 and 1.58 , we take $\alpha_{\Lambda}=3 / 2$ as a guide when making approximations in this section. In the next section, we will use WMAP measurements of $h$ and $\Omega_{\Lambda}$ to determine $\alpha_{\Lambda}$.

\subsection{The model galaxy}

A number of geometries have been used to model the formation of galaxies [15-17], including a spherical geometry. Because we will be making connection with cosmology, we are interested in the large-distance properties of the density profile, and at such distances, detail structures of galaxies are washed out; only the spherically symmetric features survive. We thus use a spherical geometry to model our idealized galaxy, and will focus on the general structure of the galaxy instead of on the details.

To determine the density profile, we divide space into the following three regions. The first, Region $\mathrm{I}=\left\{r \mid r \leq r_{H}\right.$, and $\left.\rho \gg \Lambda_{\mathrm{DE}} / 2 \pi\right\}$, encompasses the galactic core with radius, $r_{H}$. Here, the density of matter is much larger than $\Lambda_{\mathrm{DE}} / 2 \pi$. The second, Region II $=\left\{r \mid r_{H}\left\langle r \leq r_{\mathrm{II}}\right.\right.$, and $\left.\rho \gg \Lambda_{\mathrm{DE}} / 2 \pi\right\}$, encompasses the region outside the core that contains stars undergoing rotational motion with constant velocity. Here, the density of matter is also greater than $\Lambda_{\mathrm{DE}} / 2 \pi$, and the region extends out to a distance of $r_{\mathrm{II}}$, which is determined by the theory. While this region of constant rotational motion is much larger than the size of the galaxy, there are no other galaxies in the model, and we find that there is a cutoff in the density at distances beyond $r_{\mathrm{II}}$. Finally, the third, Region III $=\left\{r \mid r_{\mathrm{II}}<r\right.$, and $\left.\rho \ll \Lambda_{\mathrm{DE}} / 2 \pi\right\}$, encompasses the region where the density of matter is less than $\Lambda_{\mathrm{DE}} / 2 \pi$. We will find that the density is exponentially small here, and we will see in the next section that this region is not physically relevant.

We assume that all the stars in the model galaxy undergo circular motion. While this is an approximation, galactic rotation curves are determined with stars that undergo this motion, and we use these curves as inputs for our analysis. With this approximation, the acceleration of each star, $\mathbf{a} \equiv \ddot{\mathbf{x}}$, is a function of $r$ only. We can then take the divergence of Eq. (7), and obtain

$$
f(r)=\rho-\chi \lambda_{\mathrm{DE}}^{2} \frac{\left\{\nabla^{2} \rho-\frac{1+\alpha_{\Lambda}}{4+8 \pi \rho / \Lambda_{\mathrm{DE}}}\left(\frac{8 \pi}{\Lambda_{\mathrm{DE}}}\right)|\nabla \rho|^{2}\right\}}{\left\{1+\left(4+\frac{8 \pi \rho}{\Lambda_{\mathrm{DE}}}\right)^{1+\alpha_{\Lambda}}\right\}}
$$

where $f(r) \equiv-\nabla \cdot \mathbf{a} / 4 \pi G$ is considered a source. Because

$$
-\nabla \cdot \mathbf{a}=\frac{1}{r^{2}} \frac{\partial}{\partial r}\left[r v(r)^{2}\right]
$$

given a velocity curve for the galaxy, $v(r)$, a can be found, and the source determined. 
While a number of different models have been used in the literature to fit the observed rotational velocity curves, we use a particularly simple idealization of the curves:

$$
v^{\text {ideal }}(r)= \begin{cases}v_{H} r / r_{H} & \text { for } r \leq r_{H} \\ v_{H} & \text { for } r \geq r_{H},\end{cases}
$$

where $v_{H}$ is the asymptotic velocity of the velocity curve. We make this idealization because our purpose is not to derive the precise density profile of a galaxy; our choice of geometry does not allow for such a derivation. Instead, our purpose is to determine the overall features of the density profile; for this purpose, $v^{\text {ideal }}(r)$ is sufficient.

While $v^{\text {ideal }}(r)$ is continuous, $f(r)$ is not. We find that

$$
f(r)= \begin{cases}\rho_{H}=3 v_{H}^{2} / 4 \pi G r_{H}^{2} & \text { for } r \leq r_{H} \\ \rho_{H} r_{H}^{2} / 3 r^{2} & \text { for } r \geq r_{H} .\end{cases}
$$

Here, $\rho_{H}$ is identified as the density of matter in the galactic core, which is a constant for our idealized velocity curve.

\subsection{The density profile of the model galaxy}

In both Regions I and II, $\rho \gg \Lambda_{\mathrm{DE}} / 2 \pi$, and Eq. (9) may be approximated as

$$
f(u)=\rho+\frac{\Lambda_{\mathrm{DE}}}{8 \pi \alpha_{\Lambda}} \nabla_{u}^{2}\left(\frac{\Lambda_{\mathrm{DE}}}{8 \pi \rho}\right)^{\alpha_{\Lambda}},
$$

where $u=r / \chi^{1 / 2} \lambda_{\mathrm{DE}}$, and $\nabla_{u}$ denotes the derivative with respect to $u$.

In Region III, on the other hand, we find that $f(r)$ is negligibly small, and can be set to zero. Equation (9) then reduces to

$$
0=\rho-\frac{1}{1+4^{1+\alpha_{\Lambda}}} \nabla_{u}^{2} \rho
$$

\subsubsection{The solution in regions I and II}

Because $f(u)=\rho_{H}$ in Region I, we find that the density, $\rho_{\mathrm{I}}(r)$, in this region is simply $\rho_{H}$.

To find the density, $\rho_{\mathrm{II}}(r)$, in Region II, asymptotic analysis [20] is used. We first find a solution to Eq. (11) in a region where $\rho_{H}\left(r_{H} / r\right)^{2} / 3 \ll \rho(r)$. This is equivalent to making the anzatz that within Region II there is a point $r_{\text {asymp }}$ beyond which the driving term in Eq. (11) is negligibly small compared to the density. Equation (11) then reduces to

$$
0=\rho_{\text {asymp }}+\frac{\Lambda_{\mathrm{DE}}}{8 \pi \alpha_{\Lambda}} \nabla_{u}^{2}\left(\frac{\Lambda_{\mathrm{DE}}}{8 \pi \rho_{\mathrm{asymp}}}\right)^{\alpha_{\Lambda}},
$$


where $\rho_{\text {asymp }}$ is the asymptotic density. We then perturb off $\rho_{\text {asymp }}$ to find the density near the galactic core.

The solution to Eq. (13) has the form

$$
\rho_{\text {asymp }}=\frac{\Lambda_{\mathrm{DE}}}{8 \pi} \Sigma\left(\alpha_{\Lambda}\right) u^{p}
$$

where $p$ and $\Sigma\left(\alpha_{\Lambda}\right)$ are solutions to

$$
0=1-\frac{p\left(1-\alpha_{\Lambda} p\right)}{\left[\Sigma\left(\alpha_{\Lambda}\right)\right]^{1+\alpha_{\Lambda}}} \frac{1}{u^{p\left(1+\alpha_{\Lambda}\right)+2}} .
$$

Thus $p=-2 /\left(1+\alpha_{\Lambda}\right)$, while $\Sigma\left(\alpha_{\Lambda}\right)$ is given by

$$
0=1+\frac{2\left(1+3 \alpha_{\Lambda}\right)}{\left(1+\alpha_{\Lambda}\right)^{2}} \frac{1}{\left[\Sigma\left(\alpha_{\Lambda}\right)\right]^{1+\alpha_{\Lambda}}} .
$$

For $\rho_{\text {asymp }}$ to be positive, $\Sigma\left(\alpha_{\Lambda}\right)>0$, and thus there must be positive solutions to Eq. (16). Such solutions exist only if $\alpha_{\Lambda}$ is the ratio of a odd integer to an even integer. Our choice of $\alpha_{\Lambda}=3 / 2$ satisfies this criteria, and we arrive at the following asymptotic solution

$$
\rho_{\mathrm{asymp}}(r)=\frac{\Lambda_{\mathrm{DE}}}{8 \pi} \Sigma\left(\alpha_{\Lambda}\right)\left(\frac{\chi \lambda_{\mathrm{DE}}^{2}}{r^{2}}\right)^{1 /\left(1+\alpha_{\Lambda}\right)}
$$

with

$$
\Sigma\left(\alpha_{\Lambda}\right)=\left[\frac{2\left(1+3 \alpha_{\Lambda}\right)}{\left(1+\alpha_{\Lambda}\right)^{2}}\right]^{1 /\left(1+\alpha_{\Lambda}\right)} .
$$

To justify our anzatz that $r_{\text {asymp }}$ lies within Region II, we set $f\left(r_{\text {asymp }}\right)=$ $\rho_{\text {asymp }}\left(r_{\text {asymp }}\right)$, and find the bound

$$
\frac{r_{\mathrm{asymp}}}{r_{H}}=\left(\frac{8 \pi \rho_{H}}{3 \Lambda_{\mathrm{DE}}} \frac{u_{H}^{2 /\left(1+\alpha_{\Lambda}\right)}}{\Sigma\left(\alpha_{\Lambda}\right)}\right)^{\left(1+\alpha_{\Lambda}\right) / 2 \alpha_{\Lambda}}
$$

where $u_{H}=r_{H} / \chi^{1 / 2} \lambda_{\mathrm{DE}}$. For $\alpha_{\Lambda}=3 / 2, \rho_{H} \sim 10^{-24} \mathrm{~g} / \mathrm{cm}^{3}$, and $r_{H}=10 \mathrm{kpc}$, $r_{\text {asymp }} \leq 6.8 r_{H}$; the anzatz is thus valid throughout the great majority of Region II for the range of galaxies we are interested in. The upper limit, $r_{\mathrm{II}}$, to Region II, on the other hand, is found by setting $8 \pi \rho_{\text {asymp }}\left(r_{\mathrm{II}}\right) / \Lambda_{\mathrm{DE}}=4$. This gives

$$
r_{\mathrm{II}}=\left[\frac{1}{4} \Sigma\left(\alpha_{\Lambda}\right)\right]^{\left(1+\alpha_{\Lambda}\right) / 2} \chi^{1 / 2} \lambda_{\mathrm{DE}}
$$

For $\alpha_{\Lambda}=3 / 2, r_{\mathrm{II}} \approx 0.27 \lambda_{\mathrm{DE}}$. 
Next, to see the structural details of the galaxy, we take $\rho_{\mathrm{II}}=\rho_{\mathrm{asymp}}+\rho_{\mathrm{II}}^{1}$. Expanding Eq. (11) to first order in $\rho_{\text {II }}^{1}$ gives

$$
\frac{2\left(1+3 \alpha_{\Lambda}\right)}{\left(1+\alpha_{\Lambda}\right)^{2}}\left[\frac{\rho_{H}}{3}\left(\frac{u_{H}}{u}\right)^{2}\right]=\frac{2\left(1+3 \alpha_{\Lambda}\right)}{\left(1+\alpha_{\Lambda}\right)^{2}} \frac{\widehat{\rho}_{\mathrm{II}}^{1}}{u^{2}}+\nabla_{u}^{2} \widehat{\rho}_{\mathrm{II}}^{1} .
$$

where $\widehat{\rho}_{\mathrm{II}}^{1}=u^{2} \rho_{\mathrm{II}}^{1}$. The particular solution to Eq. (21) is again the constant solution, but now for $\widehat{\rho}_{\mathrm{II}}^{1}$; this corresponds to $\rho_{\mathrm{II}}^{1}=\rho_{H} r_{H}^{2} / 3 r^{2}$. The solution of the homogeneous equation is straightforward, and combined with $\rho_{\text {asymp }}$ from Eq. (17), we arrive at the density profile in Region II

$$
\begin{aligned}
\rho_{\mathrm{II}}(r)= & \frac{\Lambda_{\mathrm{DE}}}{8 \pi} \Sigma\left(\alpha_{\Lambda}\right)\left(\frac{\chi \lambda_{\mathrm{DE}}^{2}}{r^{2}}\right)^{1 /\left(1+\alpha_{\Lambda}\right)}+\frac{1}{3} \rho_{H}\left(\frac{r_{H}}{r}\right)^{2} \\
& +\left(\frac{r_{H}}{r}\right)^{5 / 2}\left(C_{\cos } \cos \left[v \log \left(\frac{r}{r_{H}}\right)\right]+C_{\sin } \sin \left[v \log \left(\frac{r}{r_{H}}\right)\right]\right) .
\end{aligned}
$$

Here $v=\left[2\left(1+3 \alpha_{\Lambda}\right) /\left(1+\alpha_{\Lambda}\right)^{2}-1 / 4\right]^{1 / 2}$, while

$$
\begin{aligned}
C_{\mathrm{cos}} & =\frac{2}{3} \rho_{H}-\rho_{\mathrm{asymp}}\left(r_{H}\right), \\
\nu C_{\mathrm{sin}} & =\frac{7}{3} \rho_{H}-\frac{1}{2} \frac{\left(1+5 \alpha_{\Lambda}\right)}{\left(1+\alpha_{\Lambda}\right)} \rho_{\mathrm{asymp}}\left(r_{H}\right),
\end{aligned}
$$

are determined by the boundary conditions for $\rho(r)$ at $r_{H}$.

The density, $\rho_{\mathrm{II}}(r)$, thus consists of the sum of two parts. The first part, $\rho_{\text {asymp }}(r)$, corresponds to a background, asymptotic density, and depends solely on Dark Energy, fundamental constants, the exponent $\alpha_{\Lambda}$, and the dimensionality of spacetime. It is universal, and has the same form irrespective of the detailed structure of the galaxy. The second part, $\rho_{\mathrm{II}}^{1}(r)$, on the other hand, does depend on the detail structure of the galaxy. Variations in $\rho_{\mathrm{II}}^{1}$ are on the scale of $r_{H}$, the core size; in contrast, variations in $\rho_{\text {asymp }}$ are on the scale of $\lambda_{\mathrm{DE}}$, the Dark Energy length scale. While our analysis is done only to first order in the perturbation of $\rho_{\mathrm{II}}$, these features of $\rho_{\mathrm{II}}^{1}$ hold to higher orders as well.

The perturbation, $\rho_{\mathrm{II}}^{1}$, itself depends on two terms. The first has a power law dependence of $\left(r_{H} / r\right)^{2}$, while the second has a power law dependence of $\left(r_{H} / r\right)^{5 / 2}$. Thus, near the galactic core $\rho_{\mathrm{II}}^{1} \sim 1 / r^{5 / 2}$, while for $r \gg r_{H}, \rho_{\mathrm{II}}^{1} \sim 1 / r^{2}$. This behavior at large $r$ is consistent with the pseudo-isothermal density profile [5] observed in spiral galaxies. Both terms in the perturbation decrease rapidly with $r$, and thus detail structural features of the galaxy disappear quickly with distance from its core. 


\subsubsection{A natural cutoff for $\rho(r)$}

With the boundary condition $\rho_{\mathrm{II}}\left(r_{\mathrm{II}}\right)=\rho_{\mathrm{III}}\left(r_{\mathrm{II}}\right)$, the only solution to Eq. (12) in Region III that is spherically symmetric and finite as $r \rightarrow \infty$ is

$$
\begin{aligned}
\rho_{\mathrm{III}}(r)= & \frac{\Lambda_{\mathrm{DE}}}{8 \pi} \Sigma\left(\alpha_{\Lambda}\right) \frac{\sqrt{\chi} \lambda_{\mathrm{DE}}}{r}\left(1+4^{1+\alpha_{\Lambda}}\right)^{\frac{1}{2} \frac{\left(1-\alpha_{\Lambda}\right)}{\left(1+\alpha_{\Lambda}\right)}} \\
& \times \exp \left(1-\frac{r}{\lambda_{\mathrm{DE}}} \sqrt{\frac{1+4^{1+\alpha_{\Lambda}}}{\chi}}\right) .
\end{aligned}
$$

In this region, the density decreases to zero exponentially fast, and the profile essentially cuts off at $\sqrt{\chi /\left(1+4^{1+\alpha_{\Lambda}}\right)} \lambda_{\mathrm{DE}}$; there is therefore a natural cutoff built within the theory. The scale of this cutoff is approximately $0.20 \lambda_{\mathrm{DE}}$ for $\alpha_{\Lambda}=3 / 2$, while from Eq. $(20), r_{\mathrm{II}} \approx 0.27 \lambda_{\mathrm{DE}}$; to a good approximation $r_{\mathrm{II}} \approx \sqrt{\chi /\left(1+4^{1+\alpha_{\Lambda}}\right)} \lambda_{\mathrm{DE}}$. Regions II and III thus overlap, and the asymptotic analysis is self-consistent. As $\sqrt{\chi /\left(1+4^{1+\alpha_{\Lambda}}\right)} \lambda_{D E}$ is a scale set by the theory as opposed to Eq. (20), which is set by an approximation scheme, we take $r_{\mathrm{II}} \equiv \sqrt{\chi /\left(1+4^{1+\alpha_{\Lambda}}\right)} \lambda_{\mathrm{DE}}$ from now on.

\section{A cosmological check}

In this section, we use the recent measurements of the Hubble constant, $h$, by WMAP to set $\alpha_{\Lambda}$. This $\alpha_{\Lambda}$ is then used to calculate $\sigma_{8}$ and $R_{200}$ using the model galaxy derived in the previous section. These calculated values are then compared with WMAP measurements for $\sigma_{8}$, and observations of $R_{200}$, thereby providing a cosmological check of the extended GEOM.

The density profile for the model galaxy in the previous section was calculated using $\eta_{\mu \nu}$ as the background metric in the nonrelativistic and weak gravity limits. While this approximation is appropriate at the galactic scale, we would not, in general, expect our $\rho(\mathbf{x})$ to be valid at cosmological scales; at one point, we would expect the local curvature of the universe to introduce corrections that were not taken into account in the original linearization of the metric, $g_{\mu \nu}$. WMAP and the Supernova Legacy Survey put $\Omega_{K}=-0.011_{ \pm 0.012}$, however, while WMAP and the HST key project set $\Omega_{K}=-0.014_{ \pm 0.017}$ [4]. Measurements have thus shown that the universe is within experimental error of being flat, and indeed, WMAP's determination of $h=0.732_{-0.032}^{+0.031}$ was made under this assumption. As the density profiles of galaxies do not increase at large distances from the galactic core, it is consistent-both internal to the perturbative analysis and external with experimental observations- to extend our solution in Eq. (22) to cosmological scales.

Like cosmological models, the spacetime considered in Sect. 2 is divided into timeslices. Also like cosmological models, the radial coordinate, $r$, used in this paper is the proper distance between two points on the same time-slice. This distance is not what is observed in astronomical observations, however. An object separated from an observer by a proper-distance can only be observable some time in the future, when 
the object enters the past lightcone of the observer. This is an important distinction that will be used when setting $\alpha_{\Lambda}$.

Our model from Sect. 2 is for a single galaxy, while the universe certainly contains more than just one galaxy. That we can apply our single-galaxy model to observations made on the universe such as those by WMAP is due to the large separations between galaxies, and the universal properties of $\rho_{\text {asymp }}$. Separations between galaxies are observed to be on the mega-parsec scale, while the core size of galaxies, $r_{H}$, are on the kilo-parsec scale. As we showed in Sect.2, the detailed structure of the model galaxy is described by $\rho_{\mathrm{II}}^{1}$, which dies off as $1 / r^{2}$ for large $r$. Much beyond $\sim 6.8 r_{H}$, knowledge of the detail structure of galaxies is washed out, and only $\rho_{\text {asymp }}$ remains. Thus, the single galaxy model in Sect. 2 is an adequate model of a galaxy in the universe when it is limited to some region centered on the galaxy, and because of the universal properties of $\rho_{\text {asymp }}$, the extent of this region can be quite large. Indeed, due to the large separation between galaxies, it would be appropriate to extend this region to the surface of a sphere of radius, $d$, where $2 d$ is the proper distance from the galaxy to its nearest neighbor galaxy. (If the density is required to be everywhere continuous, the shape of this region can be adjusted to ensure that this requirement is met.) The profile of our model galaxy is applicable within this sphere. Moreover, we are able to describe a distribution of many galaxies in the universe by replicating the single-galaxy model; the location and parameters of the replicated model need only be changed to reflect the properties of each galaxy in the distribution.

\section{$4.1 \alpha_{\Lambda}$ and the Hubble length, $\lambda_{H}$}

The density profile for the model galaxy in Sect. 2 decreases exponentially to zero beyond a distance $r_{\text {II }}$ from the center of the galaxy. As $r_{\text {II }}$ does not depend on the detailed structure of galaxies, and because a precipitous decrease in the local density of matter has not been observed in the universe, we argue below that we must have $d<r_{\text {II }}$ between any two galaxies in our distribution of galaxies. This restriction determines $r_{\text {II }}$, which in turn will determine $\alpha_{\Lambda}$.

Consider a two spiral galaxy model where the two galaxies are separated by a distance $2 d$; galaxy A is located at $\mathbf{d}$, while galaxy B is located at $-\mathbf{d}$. We require that $d$ be much larger than the size of either galaxy. We also require that while the two galaxies may move relative to each other, this motion takes place over a very long time scale, and thus $\mathbf{d}$ can approximated as a constant independent of time. This is a restrictive requirement; observations show that galaxies do orbit one another, and there can be significant relative motion of the galaxies. We do not need to construct a detail model of the dynamics of galaxies - or even the density profile of them - to set a value for $\alpha_{\Lambda}$, however. All that is required is to determine the maximum separation between galaxies within the theory, and then to compare this length with the proper-distance between galaxies in cosmological models. This can be done within a model where the two galaxies do not move relative to one another.

As with the single galaxy case, we approximate the motion of stars in both galaxies as undergoing circular motion about the center of each galaxy. Since the separation between galaxies is much larger than the size of galaxies, the motion of stars within 
one galaxy does not affect the motion of stars within the other. Thus, Eq. (11) still holds near the core of galaxy A after replacing $f(\mathbf{x})$ by $f^{A}(\mathbf{x}-\mathbf{d})$. It also holds near the core of galaxy B after replacing $f(\mathbf{x})$ by $f^{B}(\mathbf{x}+\mathbf{d})$. Here, $f^{A}$ and $f^{B}$ have the same form as $f(\mathbf{x})$ given by $v^{\text {ideal }}(r)$, but with $r$ replaced by $|\mathbf{x} \pm \mathbf{d}|$, and with $r_{H}$ and $v_{H}$ replaced by the core size and asymptotic rotational velocity of the respective galaxy.

While we can, at least in principle, follow the same analysis as before to determine the density profile of the galaxies - the core of the two galaxies are well separated from one another-this detailed analysis is not needed to determine $\alpha_{\Lambda}$. Instead, we note that as in the single galaxy case, spacetime can be divided into two domains, $\mathfrak{D}$ and $\mathfrak{Z}$. In the first, $\mathfrak{D}, \rho>>\Lambda_{\mathrm{DE}} / 2 \pi$, and asymptotic analysis can still be used to determine the density profile of the galaxies as $\rho(\mathbf{x})=\rho_{\text {asymp }}(\mathbf{x})+\rho_{\mathrm{II}}^{1 A}(\mathbf{x}-\mathbf{d})+\rho_{\mathrm{II}}^{1 B}(\mathbf{x}+\mathbf{d})$. Here, $\rho_{\text {asymp }}$ is a solution of Eq. (13), but now in the presence of two galaxies instead of one. (While the presence of the galaxies does not explicitly appear in this equation, the locations of the galaxies determine the boundary conditions for $\rho$, and thus establish the underlying geometry as being axially symmetric.) As before, $\rho_{\mathrm{II}}^{1 A}(\mathbf{x}-\mathbf{d})$ and $\rho_{\mathrm{II}}^{1 B}(\mathbf{x}+\mathbf{d})$ are the first-order perturbations off $\rho_{\text {asymp }}(\mathbf{x})$ near the core of each galaxy; they establish the structure of each. Because of the large separation between galaxies, $\rho_{\mathrm{II}}^{1 A}$ and $\rho_{\mathrm{II}}^{1 B}$ can be solved independently using the equivalent of Eq. (21) for the particular $\rho_{\text {asymp }}$ obtained for the two galaxy model, and with the origin shifted to the center of each galaxy.

In the second, $\mathfrak{Z}, \rho<<\Lambda_{\mathrm{DE}} / 2 \pi$, and in this domain, Eq. (12) holds. As such, the density decreases exponentially to zero, and for most of the region, we may take $\rho \approx 0$. Note that this behavior of $\rho$ is set solely by the form of Eq. (12), and does not depend on either the distribution of the galaxies, or the detail structure of them.

Consider now the behavior of the density profile along the line connecting the cores of the two galaxies. If the separation, $2 d$, between the two galaxies is smaller than some distance $2 d_{\max }$, then for all points along this line $\rho>\Lambda_{\mathrm{DE}} / 2 \pi$. The density, $\rho(\mathbf{x})$, is locally maximum at the core, $\mathbf{x}=-\mathbf{d}$, of galaxy $\mathrm{B}$, and decreases as the distance, $|\mathbf{x}+\mathbf{d}|$, from the core increases. Since Eq. (13) does not depend on the detail structure of either galaxy, the density reaches a local minimum, $\rho_{\min } \equiv \rho(0)$, at the midpoint between the two galaxies. The density then increases as the distance increases until it is again a local maximum at the core, $\mathbf{x}=\mathbf{d}$, of galaxy A. When $d<d_{\max }, \rho_{\min }>\Lambda_{\mathrm{DE}} / 2 \pi$, and in this case $\mathfrak{D}$ is simply connected.

To estimate the scale of $d_{\max }$, consider the limit of large $d$. As the separation between galaxies increases, $\rho_{\min }$ decreases until at one point $\rho_{\min }<<\Lambda_{\mathrm{DE}} / 2 \pi$. When this happens, the midpoint between the galaxies is contained in $\mathfrak{Z}$, and $\mathfrak{D}$ is no longer simply connected. Instead, $\mathfrak{D}=\mathfrak{D}_{A} \cup \mathfrak{D}_{B}$, where $\mathfrak{D}_{A}$ and $\mathfrak{D}_{B}$ contains the core of galaxy A and galaxy $\mathrm{B}$, respectively, and $\mathfrak{D}_{A} \cap \mathfrak{D}_{B}=\emptyset$. The two galaxies are separated by a region in $\mathfrak{Z}$ where $\rho$ decreases exponentially fast. As such, for large enough separations the density profile of one galaxy will not depend on the presence of the other, and we will be back to the single galaxy case. For each galaxy, $\rho$ is now given by Eq. (22), with $r$ measured from the center of the galaxy, and the $r_{H}$ and $v_{H}$ set by the properties of this galaxy. In this limit, $\mathfrak{D}_{A}$ and $\mathfrak{D}_{B}$ are spheres centered at the core of their respective galaxy, and each has radii $r_{\mathrm{II}}$. Since $2 d_{\max }$ is the maximum 
separation between galaxies for which $\mathfrak{D}$ is simply connected, we estimate $d_{\max }=$ $r_{\mathrm{II}}$.

As astronomical observations have not shown that the density of matter decreases precipitously at any point along the separation two galaxies, we require that in our model of a distribution of galaxies, the separation between galaxies must be less than $2 r_{\text {II }}$.

Since $2 r_{\text {II }}$ is the maximum possible proper distance between galaxies in the model, it must be cosmological in scale. It would be physically appropriate to identify this scale with $\lambda_{e H}$ - the proper distance to the cosmological event horizon at present day-for the following reasons. Consider an observer on galaxy A, which at the present day, $t_{0}$, is separated from galaxy $\mathrm{B}$ by a proper distance $r_{\mathrm{II}}$. If $2 r_{\mathrm{II}}<\lambda_{e H}$, at a finite time, $t$, in the future galaxy B will pass into the past lightcone of galaxy $\mathrm{A}$, and thus be observed by the observer on A. If, on the other hand, $2 r_{\mathrm{II}}>\lambda_{e H}$, then for no time $t$ will galaxy B pass within the pass lightcone of galaxy A, and will never be observed by the observer on A. More importantly, when $2 r_{I I}>\lambda_{e H}$, no physical property of galaxy A can be influenced by galaxy B in the future, and thus the presence of galaxy B has no physical significance to the observer on A. Galaxy A and galaxy B will be causally disconnected.

While these are good, physical reasons for identifying $2 r_{\text {II }}$ with $\lambda_{e H}$, it is not physically possible to measure $\lambda_{e H}$ directly. All physical measurements are made on objects within or on the past lightcone of an observer. Because $\lambda_{e H}$ is the proper distance to the event horizon at present day, it cannot, even in principle, be measured; it is at best inferred. What is physically measurable, on the other hand, is $\lambda_{H}$, which is also the radius of the Hubble sphere. Although it is shown in [21] that for a $\Lambda$ CDM model with $\left(\Omega_{M}, \Omega_{\Lambda}\right)=(0.3,0.7)$ and $h=0.70$ the cosmological event horizon is not equivalent to the Hubble sphere, $1 / H$ is the time it has taken galaxies to achieve their current separations [14]. The Hubble scale is the maximum distance that light has traveled in this time. Thus, $\lambda_{H}$ is the maximum separation between galaxy $\mathrm{A}$ and galaxy $\mathrm{B}$ from which an observer on one could have observed the formation of the other at some time in the past; it would be possible for the formation of one galaxy can influence the formation of the other.

There are thus also good physical reasons for identifying $2 r_{\mathrm{II}}$ with $\lambda_{H}$. As the difference between $\lambda_{e H}$ and $\lambda_{H}$ calculated in [21] is very small at present day, and because $\lambda_{H}$ is a natural length scale in cosmology that is explicitly measurable, we identified $2 r_{H}=\lambda_{H}$. Since we can express

$$
r_{I I}=\left[\frac{8 \pi \chi}{3 \Omega_{\Lambda}\left(1+4^{1+\alpha_{\Lambda}}\right)}\right]^{1 / 2} \lambda_{H},
$$

this identification sets the value for $\alpha_{\Lambda}$. Here, $\Omega_{\Lambda}=0.716_{ \pm 0.055}$ from [4] is the fractional density of Dark Energy in the universe. The solution to Eq. (25) gives $\alpha_{\Lambda}=1.56_{ \pm 0.10}$, which is close to the working value of $3 / 2$ for $\alpha_{\Lambda}$ used in the previous section. Note that to the lowest significant figure, this $\alpha_{\Lambda}$ is the ratio of an odd to an even integer as well. 
$4.2 \sigma_{8}, R_{200}$, and observational data for the galactic rotation curves

Following [14] and [22], linear fluctuations in the density within a sphere, $S_{8}$, of radius $8 h^{-1} \mathrm{Mpc}$ are characterized by

$$
\delta(\mathbf{x})=\frac{\rho(\mathbf{x})-\langle\rho\rangle_{S_{8}}}{\langle\rho\rangle_{S_{8}}},
$$

where $\langle\cdots\rangle_{S_{8}}$ denotes the spatial-average over $S_{8}$. Of particular interest is $\sigma_{8}$, the rms fluctuations of $\delta$ within $S_{8}$,

$$
\sigma_{8}^{2} \equiv\left\langle\left(\delta(\mathbf{x})^{2}\right)\right\rangle_{S_{8}}
$$

Since $\sigma_{8}$ is used to study the formation of structure in the universe, usual calculations of $\sigma_{8}$ involves modeling the evolution of density fluctuations in the universe as it expands. These calculations are done in Fourier space, and $\sigma_{8}$ is usually expressed in terms of a power spectrum, $\Delta_{k}$, and a window function, $W(k)(W(r)$ in real space), that restricts the fluctuations in the density to the region $S_{8}$. There are a number of subtleties in this calculation, however, and in particular, care must be taken in choosing $W(k)$. Indeed, the simplest choice

$$
W(r)= \begin{cases}1 & \text { for } r \leq 8 h^{-1} \mathrm{Mpc} \\ 0 & \text { for } r>8 h^{-1} \mathrm{Mpc}\end{cases}
$$

called the top hat window, produces a hard cutoff at $r=8 h^{-1} \mathrm{Mpc}$, and thus can introduce spurious fluctuations at scales $k \sim h / 8 \mathrm{Mpc}^{-1}$.

Our focus is not on the formation of structure during the expansion of the universe, however. It is on using $\sigma_{8}$ to test the predictions of the extended GEOM. As we have an explicit density profile for a model galaxy, we can calculate $\sigma_{8}$ directly from its definition, Eq. (27), using the top-hat window function; a power spectrum is not needed in our calculation.

One estimate of the characteristic length scale for the galaxy-galaxy correlation function [22] is $5 h^{-1}$; thus there is roughly one galaxy within $S_{8}$. Consequently, we calculate $\sigma_{8}$ with the approximation that there is a single galaxy in $S_{8}$ with its core located at the center of the sphere, and with a specific set of values, $v_{H}^{*}$ and $r_{H}^{*}$, for the asymptotic velocity and core radius of this galaxy.

As $\rho(r)$ varies significantly across $S_{8}$, we begin by finding the average density,

$$
\begin{aligned}
\langle\rho\rangle_{8} & \equiv\left\langle\rho_{H} \theta\left(r_{H}-r\right)\right\rangle_{8}+\left\langle\rho_{\text {asymp }}(r) \theta\left(r-r_{H}\right)\right\rangle_{8}+\left\langle\rho_{\mathrm{II}}^{1}(r) \theta\left(r-r_{H}\right)\right\rangle_{8}, \\
& =3\left(\frac{1+\alpha_{\Lambda}}{1+3 \alpha_{\Lambda}}\right) \rho_{\text {asymp }}\left(u_{8}\right) D\left(u_{8}\right)
\end{aligned}
$$


in $S_{8}$. Here, $\theta(x)$ is the step function, and $u_{8}=8 h^{-1} \mathrm{Mpc} / \chi^{1 / 2} \lambda_{D E}$. The resultant average density is proportional to $\rho_{\text {asymp }}\left(u_{8}\right)$, with

$$
\begin{aligned}
D\left(u_{8}\right) \equiv & 1-y_{8}^{\frac{1+3 \alpha_{\Lambda}}{1+\alpha_{\Lambda}}}+\left(\frac{1+3 \alpha_{\Lambda}}{1+\alpha_{\Lambda}}\right) \zeta-\frac{3}{2}\left(1+\alpha_{\Lambda}\right)\left[\frac{1}{2} \widetilde{C}_{\cos }-v \widetilde{C}_{\sin }\right] y_{8} \\
+\frac{3}{2}\left(1+\alpha_{\Lambda}\right) y_{8}^{1 / 2}( & {\left[\frac{1}{2} \widetilde{C}_{\cos }-v \widetilde{C}_{\sin }\right] \cos \left[v \log y_{8}\right] } \\
& \left.-\left[\frac{1}{2} \widetilde{C}_{\sin }+v \widetilde{C}_{\cos }\right] \sin \left[v \log y_{8}\right]\right) .
\end{aligned}
$$

Here, $y_{8}=r_{H} /\left(8 h^{-1}\right)$, and

$$
\widetilde{C}_{\cos } \equiv \frac{2}{3} \zeta-\frac{1}{3} y_{8}^{2 \alpha_{\Lambda} /\left(1+\alpha_{\Lambda}\right)}, \quad \nu \widetilde{C}_{\sin } \equiv \frac{7}{3} \zeta-\frac{1}{6}\left(\frac{1+5 \alpha_{\Lambda}}{1+\alpha_{\Lambda}}\right) y_{8}^{2 \alpha_{\Lambda} /\left(1+\alpha_{\Lambda}\right)}
$$

The parameter

$$
\zeta=\frac{2 u_{8}^{-2 \alpha_{\Lambda} /\left(1+\alpha_{\Lambda}\right)}}{\Sigma\left(\alpha_{\Lambda}\right) \chi\left(\alpha_{\Lambda}\right)}\left(\frac{v_{H}^{*}}{c}\right)^{2}
$$

depends explicitly on the detail structure of the galaxy. As $\zeta \sim 4 \times 10^{-3}$ for $v_{H}^{*}=$ $200 \mathrm{~km} / \mathrm{s}, D\left(u_{8}\right) \approx 1$, and the asymptotic density dominates $\langle\rho\rangle_{8}$, not the detail structure of the galaxy.

This is not the case for $\left\langle\delta(\mathbf{x})^{2}\right\rangle_{8}$, which involves the integration of $\rho^{2}(r)$ over $S_{8}$. Not surprising, it is now the behavior of the density near the core that is relevant. Indeed, we find

$$
\begin{aligned}
\sigma_{8}^{2}= & -1+\frac{1}{\left[D\left(u_{8}\right)\right]^{2}}\left[\frac{\left(1+3 \alpha_{\Lambda}\right)}{\left(1+\alpha_{\Lambda}\right)}\right]^{2}\left(\frac{\zeta^{2}}{3}\left[\frac{4}{y_{8}}-1\right]\right. \\
& +\frac{1}{3}\left[\frac{\alpha_{\Lambda}+1}{3 \alpha_{\Lambda}-1}\right]\left[1-y_{8}^{\frac{3 \alpha_{\Lambda}-1}{\alpha_{\Lambda}+1}}\right]+\frac{2 \zeta}{3}\left(\frac{\alpha_{\Lambda}+1}{\alpha_{\Lambda}-1}\right)\left(1-y_{8}^{\frac{\alpha_{\Lambda}-1}{\alpha_{\Lambda}+1}}\right) \\
& -\frac{4\left(\alpha_{\Lambda}-3\right)\left(\alpha_{\Lambda}+1\right)}{\left(\alpha_{\Lambda}-3\right)^{2}+4 v^{2}\left(1+\alpha_{\Lambda}\right)^{2}}\left\{\left[\widetilde{C}_{\cos }-\frac{2\left(1+\alpha_{\Lambda}\right)}{\left(\alpha_{\Lambda}-3\right)} v \widetilde{C}_{\sin }\right] y_{8}^{\frac{\alpha_{\Lambda}-1}{\alpha_{\Lambda}+1}}\right. \\
& -y_{8}^{1 / 2}\left(\left[\widetilde{C}_{\cos }-\frac{2\left(1+\alpha_{\Lambda}\right)}{\left(\alpha_{\Lambda}-3\right)} v \widetilde{C}_{\sin }\right] \cos \left[v \log y_{8}\right]\right. \\
& \left.\left.-\left[\widetilde{C}_{\sin }+\frac{2\left(1+\alpha_{\Lambda}\right)}{\left(\alpha_{\Lambda}-3\right)} v \widetilde{C}_{\cos }\right] \sin \left[v \log y_{8}\right]\right)\right\} \\
& +\frac{3 \zeta}{y_{8}} \frac{1}{9 / 4+v^{2}}\left\{\left[\widetilde{C}_{\cos }+\frac{2 v}{3} \widetilde{C}_{\sin }\right]-y_{8}^{\frac{3}{2}}\left(\left[\widetilde{C}_{\cos }+\frac{2 v}{3} \widetilde{C}_{\sin }\right] \cos \left[v \log y_{8}\right]\right.\right. \\
& \left.\left.+\left[\frac{2 v}{3} \widetilde{C}_{\cos }-\widetilde{C}_{\sin }\right] \sin \left[v \log y_{8}\right]\right)\right\}
\end{aligned}
$$




$$
\begin{aligned}
& +\frac{3}{4}\left[\frac{1}{y_{8}}-y_{8}\right]\left[\widetilde{C}_{\cos }^{2}+\widetilde{C}_{\sin }^{2}\right]+\frac{3}{4\left(1+v^{2}\right) y_{8}}\left[\widetilde{C}_{\cos }^{2}-\widetilde{C}_{\sin }^{2}+2 v \widetilde{C}_{\cos } \widetilde{C}_{\sin }\right] \\
& -\frac{3 y_{8}}{4\left(1+v^{2}\right)}\left\{\left[\widetilde{C}_{\cos }^{2}-\widetilde{C}_{\sin }^{2}+2 v \widetilde{C}_{\cos } \widetilde{C}_{\sin }\right] \cos \left[2 v \log y_{8}\right]\right. \\
& \left.\left.+\left[v\left(\widetilde{C}_{\cos }^{2}-\widetilde{C}_{\sin }^{2}\right)-2 \widetilde{C}_{\cos } \widetilde{C}_{\sin }\right] \sin \left[2 v \log y_{8}\right]\right\}\right) .
\end{aligned}
$$

To obtain values for $v_{H}^{*}$ and $r_{H}^{*}$, we are guided by the operational definition of $\sigma_{8}$ described in [22]. This definition involves choosing a $S_{8}$ centered at a given point in the sky, calculating the average mass in it, shifting this center to another point on the sky, repeating the measurement, and continuing until all points in the sky is, in principle, covered. The set of all such averages then forms an ensemble of such measurements, and the rms fluctuation in the mass can then be calculated for this ensemble. ${ }^{1}$

Consistent with this operational definition of $\sigma_{8}, v_{H}^{*}$ and $r_{H}^{*}$ should be for a representative spiral galaxy for the universe. Such a galaxy would in principle be found through a survey of spiral galaxies, which would result in an ensemble of asymptotic rotation velocities and core radii for the galaxies in the universe. An average for each parameter can then be taken, and identified with $v_{H}^{*}$ and $r_{H}^{*}$, which in turn can be used to construct the representative galaxy. While such a survey has not yet been done, there exists in the literature a large repository of measurements of galactic rotational velocity curves and core radii [5-13]. Taken as a whole, these 1,393 galaxies are reasonably random, and is likely representative of the observed universe at large.

Although there have been a many studies of galactic rotation curves in the literature, what is needed here is both the rotational velocity and the core radius of galaxies. This requires both a measurement of the velocity as a function of the distance from the center of the galaxy, and a fit of the data to some model of the velocity curve. To our knowledge, this analysis has been done in four places in the literature. (The study [23] gives fits to MOND rotation curves, but does not list values for $r_{H}$.) While each of the data sets were obtained with similar physical techniques, there are distinct differences in their selection of galaxies, in the exact experimental techniques used, and in the models to which the observed rotation curves are fitted. In fact, the Hubble constant used by each is often different from one another, and from the value of $73.2 \mathrm{~km} \mathrm{~s}^{-1} \mathrm{Mpc}^{-1}$ given by WMAP. The reader is referred to the specific papers for details on how these observations were made.

A number of models are in use in the literature to fit the observed galactic rotation curves, and they all require at least two parameters to model observations. Four different models of these velocity curves are fitted to the data sets we use here, and out of these, two of them can be idealized using $v^{\text {ideal }}(r)$. For these two models there is a one-to-one correspondence between the parameters used for the fit with $v_{H}$ and $r_{H}$. Data from these fits are then averaged to determine $v_{H}^{*}$ and $r_{H}^{*}$.

\footnotetext{
1 Measurements of the average mass in $S_{8}$ are necessarily done at different times. As such, a Markovian assumption must be made that the measurements of the average mass over time is equivalent to an ensemble of average masses made at equal times. This same assumption must be made when determining the representative galaxy.
} 
A summary of the data sets, and how $v_{H}$ and $r_{H}$ are obtained for each are as follows:

de Blok et al. Data Set De Blok and coworkers made detailed measurements of 60 LSB galaxies [7], and fits of the pseudo-isothermal velocity curve were done for 30 of them [5]. Later, another set of measurements of 26 LSB galaxies were made by de Blok and Bosma [6], of which 24 are different from the 30 listed in [5]. Both the data for the 30 original galaxies, and the 24 subsequent galaxies are used here. Although the authors used various models for determining the mass-to-light ratio in their measurements, we will use the data that comes from the minimum disk model, as this was the one model used for all of the galaxies in this set.

De Blok and coworkers were chiefly concern with modeling the density profiles of observed galaxies, and as such, a parameter for the profile, $\rho_{H}$, along with a parameter for the core radius, $R_{C}$, were used by them. The asymptotic value for their rotational velocity is $\sqrt{4 \pi G \rho_{H} R_{C}^{2}}$. Identifying this expression with $v_{H}$, and $\sqrt{3} R_{C}$ with $r_{H}$, we are able to extract from the de Blok data sets values for $v_{H}$ and $r_{H}$ along with their standard error. The authors used a value of $75 \mathrm{~km} \mathrm{~s}^{-1} \mathrm{Mpc}^{-1}$ for the Hubble constant.

CF Data Set In [12], Courteau presented observations of the rotational velocity curves for over 300 northern Sb-Sc UGC galaxies, and determined $r_{H}$ for each by fitting the curves to three different models of the velocity, one of which, $v^{\mathrm{vcA}}(r)=$ $\left(2 v_{C} / \pi\right) \arctan \left(r / r_{t}\right)$, is similar to the velocity curve for the pseudo-isothermal profile used by de Blok and coworkers. Like the pseudo-isothermal curve, $v^{\mathrm{vcA}}(r)$ can be approximated by the idealized velocity curve used here. In the limit $r \gg r_{t}, v^{\mathrm{vcA}} \approx$ $v_{C}$, which sets $v_{C}=v_{H}$. In the limit $r \ll r_{t}, v^{\mathrm{vcA}} \approx v_{C}\left(2 r / \pi r_{t}\right)$, which sets $r_{t}=2 r_{H} / \pi$.

A fit of these observations was also made to a velocity curve where the steepness of the transition from the hub and the asymptotic velocity curves could also be taken into account. The model curve has a form in the $r \rightarrow 0$ limit that not only disagrees with our idealized profile in one specific case, this curve does not fit all the galaxies analyzed by Courteau. We therefore did not use data from this fit.

Values for $v_{C}$ and $r_{t}$ for 351 galaxies was obtained through the VizieR service (http://vizier.u-strasbg.fr/viz-bin/VizieR). The great majority of the rotation velocity curves were based on single observations of the galaxy; only 75 of these galaxies were measured multiple times, with the majority of these galaxies being observed twice. The data set reposited at VizieR contained these multiple measurements, and we have averaged the value of $v_{C}$ and $r_{t}$ for the galaxy when multiple measurements were done. The standard error in the repeated measurements of a single galaxy can be extremely large; this was recognized in [12]. A value of $70 \mathrm{~km} \mathrm{~s}^{-1} \mathrm{Mpc}^{-1}$ was used for the Hubble constant by the author.

Mathewson et al. Data Set In [13], a survey of the velocity curves of 1,355 spiral galaxies in the southern sky was reported. Later, the rotation velocity curves for these observations were derived in [18] after folding, deprojecting, and smoothing the Mathewson data. Each of these velocity curves are due to a single observation. Courteau performed a fit of Mathewson's observations to the $v^{v c A}$ curve for 958 of the 
galaxies in [12] using a Hubble constant of $70 \mathrm{~km} \mathrm{~s}^{-1} \mathrm{Mpc}^{-1}$. The results of Courteau's analysis is reposited in VizieR as well.

Persic et al. has proposed the Universal Rotation Curve (URC) [18], [19], which has been used by them to analyze the Mathewson data. In addition, this model was the third model used by Courteau in [12] to fit both his and Mathewson's data. While the URC asymptotically approaches a constant velocity, at small $r$ the URC has a $r^{0.66}$ behavior, which is different from the pseudo-isothermal curve, the $v^{\mathrm{vcA}}$ curve, and the idealized velocity curve considered here. Although the URC has a different power-law behavior at small $r$, the difference is small enough that it is unknown how $\sigma_{8}$ will change if the URC is used in its calculation instead of the $v^{\text {ideal }}$ curve used here. We leave this for future research; for our current purposes, we did not consider fits to this velocity curve.

Rubin et al. Data Set In the early 1980s, Rubin and coworkers [8-11] presented a detailed study of the rotation curves of $16 \mathrm{Sa}, 23 \mathrm{Sb}$, and $21 \mathrm{Sc}$ galaxies. This was not a random sampling of such galaxies. Rather, these galaxies were deliberately chosen to span a specified range of $\mathrm{Sa}, \mathrm{Sb}$, and Sc galaxies, and as stated in [10], averaging values of the properties of the galaxies in this data set would have little meaning. These measurements can contribute to a combined data set of all four measurements, however, and we have included them in our analysis. While values for the core radii were not given, measurements of the rotational velocity as a function of the distance to the center of the galaxy were; we are able to fit this data to the same pseudo-isothermal rotation curve used by de Blok et al. Results of this fit is given in Appendix A. A Hubble constant of $50 \mathrm{~km} / \mathrm{s} / \mathrm{Mpc}$ was used by the authors.

Wanting to be as unbiased and as inclusive as possible, we have deliberately not culled through the data sets to select the cleanest of the rotation curves. Nevertheless, we have had to remove the data for 27 galaxies from the data sets. A list of these galaxies and the reason why they were removed are given in Appendix B, where we have also listed any peculiarities of the four base data sets.

While $v_{H}$ is easily identified for all four data sets, determining $r_{H}$ is more complicated. For the de Blok et al. data set, published values of $R_{C}$ was first scaled by $75 / 73.2$ to account for differences in the Hubble constant; $r_{H}$ is then obtained using $r_{H}=\sqrt{3} R_{C}$. The same calculation was made using the calculated values of $R_{C}$ from Appendix A, but with 50/73.2 instead of 75/73.2 to account for differences in Hubble constants. For the CF and Mathewson et al. data sets, published values of $r_{t}$ are first scaled by 70/73.2 to account for differences in Hubble constants, and $r_{H}$ is then obtained through $r_{H}=\pi r_{t} / 2$.

The values of $v_{H}^{*}$ and $r_{H}^{*}$ are then calculated for three of the four basic data sets. As the Rubin et al. data set was not random, $v_{H}^{*}$ and $r_{H}^{*}$ was not separately calculated for this data set. These measurements were instead included with data from the other three sets to form a Combined data set, and $v_{H}^{*}$ and $r_{H}^{*}$ were calculated for this set as well. The results of these calculations are giving in Table 1 . These values for $v_{H}^{*}$ and $r_{H}^{*}$ were then used to calculate $\sigma_{8}$ using Eq. (32), and the results of this calculation are given in this table as well. All the data sets give a value for $\sigma_{8}$ that agree with the WMAP value at the $95 \%$ confidence level. Moreover, the values for $\sigma_{8}$ for all of these 
Table 1 The $v_{H}^{*}(\mathrm{~km} / \mathrm{s})$ and $r_{H}^{*}(\mathrm{kpc})$ is listed for each data set along with the resultant $R_{200}(\mathrm{kpc})$ and $\sigma_{8}$

\begin{tabular}{llllc}
\hline Data Set & \multicolumn{1}{l}{$v_{H}^{*}$} & \multicolumn{1}{c}{$r_{H}^{*}$} & \multicolumn{1}{l}{$R_{200}$} & \multicolumn{1}{c}{$\sigma_{8}$} \\
\hline de Blok et al. (53) & $119.0 \pm 6.8$ & $3.62 \pm 0.33$ & $210 \pm 110$ & $0.65 \pm 0.11$ \\
CF (348) & $179.1 \pm 2.9$ & $7.43 \pm 0.35$ & $182 \pm 84$ & $0.92 \pm 0.18$ \\
Mathewson et al. (935) & $169.5 \pm 1.9$ & $15.19 \pm 0.42$ & $224 \pm 50$ & $0.654 \pm 0.093$ \\
Combined (1393) & $172.1 \pm 1.6$ & $11.82 \pm 0.30$ & $206 \pm 53$ & $0.73 \pm 0.12$ \\
\hline
\end{tabular}

The number of data points in each data set is listed in parentheses

data sets—calculated from four sets of observations taken over a 30-year span-agree with one another at the $95 \%$ confidence level as well. This is consistent with our expectation that the sample of 1,393 measurements of galactic velocity curves used here is reasonably random and unbiased.

With $v_{H}^{*}$ and $r_{H}^{*}$, it is possible to calculate $R_{200}$ by taking in Eq. (28) $u_{8} \rightarrow u_{200}=$ $R_{200} / \chi^{1 / 2} \lambda_{\mathrm{DE}}$ and $\langle\rho\rangle_{8}=200 \rho_{c}$ where the critical density, $\rho_{c}=1.006_{-0.088}^{+0.085} \times$ $10^{-29} \mathrm{~g} / \mathrm{cm}^{3}$ from [4]. The resultant equation is solved numerically for $R_{200}$; the results of this analysis is given in Table 1 as well. The range of values for $R_{200}$-with $R_{200}=$ $206_{ \pm 53} \mathrm{kpc}$ for the Combined data set-is consistent with observations. Notice that like the values for $\sigma_{8}$, the values of $R_{200}$ for these data sets also agree with one another at the $95 \%$ confidence level.

For both $\sigma_{8}$ and $R_{200}$, over $90 \%$ of the error is due to the error in $\alpha_{\Lambda}$; this is true for all the data sets. The values of $\sigma_{8}$ and $R_{200}$ are thus sensitively dependent on $\alpha_{\Lambda}$. On the one hand, this sensitivity is consistent with $\alpha_{\Lambda}$ being a power-law exponent. On the other hand, the excellent agreement between our calculated values of $\sigma_{8}$ and the WMAP value for $\sigma_{8}$ is all the more compelling because of it.

\section{Dynamics of test particles in the model galaxy}

\subsection{The extended and gravitational potentials}

Note that Eq. (5) may be written in terms of an effective potential, $\mathfrak{V}(\mathbf{x})$, as $\ddot{\mathbf{x}}=-\nabla \mathfrak{V}$, where

$$
\mathfrak{V}(\mathbf{x})=\Phi(\mathbf{x})+c^{2} \log \mathfrak{R}\left[4+8 \pi \rho / \Lambda_{\mathrm{DE}}\right] .
$$

Because of the additional terms from the extension of the GEOM on the right hand side of Eq. (5), it is not the gravitational potential, $\Phi(\mathbf{x})$, that determines the dynamics of massive particles; it is instead $\mathfrak{V}(\mathbf{x})$. This is an important distinction. Our extension of the GEOM can drastically change the metric, and these changes will have broad implications if it is $\Phi(\mathbf{x})$ and not $\mathfrak{V}(\mathbf{x})$ that determine the dynamics. To see this, we calculate explicitly $\Phi(\mathbf{x})$ in Regions I and II. In Region III, $r>r_{\text {II }}$, and motion in this region is not physically relevant.

Integrating $\nabla^{2} \Phi=4 \pi G \rho$ in Region I gives

$$
\Phi(r)=\Phi(0)+\frac{1}{2} v_{H}^{2}\left(\frac{r}{r_{H}}\right)^{2},
$$


where $\Phi(0)$ is an overall integration constant. That this is the usual expression for the Newtonian potential can be seen from the relation $\rho_{H}=3 v_{H}^{2} / 4 \pi G r_{H}^{2}$. For Region II, we find

$$
\begin{aligned}
\Phi(r)= & \Phi(0)+\frac{1}{2} v_{H}^{2}+v_{H}^{2} \log \left[\frac{r}{r_{h}}\right] \\
& -\frac{\left(1+\alpha_{\Lambda}\right)^{2}}{2\left(1+3 \alpha_{\Lambda}\right)}\left(1-\frac{r_{H}}{r}\right)\left(c^{2} \chi \Sigma\left(\alpha_{\Lambda}\right) u_{H}^{\frac{2 \alpha_{\Lambda}}{\left(1+\alpha_{\Lambda}\right)}}-6 v_{H}^{2}\right) \\
& +\frac{\left(1+\alpha_{\Lambda}\right)^{2}}{\left(1+3 \alpha_{\Lambda}\right)} \frac{c^{2} \chi}{4 \alpha_{\Lambda}}\left\{\frac{r^{2}}{\chi \lambda_{\mathrm{DE}}^{2}}\left(\frac{8 \pi \rho_{\mathrm{asymp}}(r)}{\Lambda_{\mathrm{DE}}}-\alpha_{\Lambda} \frac{8 \pi \rho_{\mathrm{II}}^{1}(r)}{\Lambda_{\mathrm{DE}}}\right)\right. \\
& \left.-\frac{r_{H}^{2}}{\chi \lambda_{\mathrm{DE}}^{2}}\left(\frac{8 \pi \rho_{\mathrm{asymp}}\left(r_{H}\right)}{\Lambda_{\mathrm{DE}}}-\alpha_{\Lambda} \frac{8 \pi \rho_{\mathrm{II}}^{1}\left(r_{H}\right)}{\Lambda_{\mathrm{DE}}}\right)\right\} .
\end{aligned}
$$

The $1 / r$ term in $\Phi(\mathbf{x})$ is expected from Newtonian gravity, and is due to the boundary conditions for $\Phi$ at $r=r_{H}$; this is true for the constant terms as well. The logarithmic term is due specifically to the source, $f(r)$, as expected. It is a long-range potential that extends out to $r_{\mathrm{II}}$, and could potentially explain the non-Newtonian interaction observed between galaxies and galactic clusters. The $\rho_{\mathrm{II}}^{1}(r) r^{2}$ term is due to the perturbation of the asymptotic density, and contains terms $\sim 1 / r^{1 / 2}$. It is due to both the boundary terms in $\rho_{\mathrm{II}}^{1}$ and the boundary conditions for $\Phi(r)$.

For the $c^{2}$ term in $\Phi(r), r^{2} \rho_{\text {asymp }} \sim r^{2 \alpha_{\Lambda} /\left(1+\alpha_{\Lambda}\right)}$, which increases as $r^{1.22}$ for $\alpha_{\Lambda}=1.56$. This would dominate the dynamics of test particles in the galaxy if the extended GEOM depended on $\Phi(\mathbf{x})$ instead of $\mathfrak{V}(\mathbf{x})$. Indeed, if the dynamics were determined by $\Phi(\mathbf{x})$, the resultant motion for stars in the galaxy would not agree with observations whatsoever. Instead, this term and the $r^{2} \rho_{\text {II }}^{1}$ term in $\Phi$ are canceled by the additional density-dependent terms in Eq. (33). To see this, in Regions I and II $\rho>>\Lambda_{\mathrm{DE}} / 2 \pi$, and we expand Eq. (33) to give

$$
\mathfrak{V}(\mathbf{x})=\Phi(0)+\frac{1}{2} v_{H}^{2}\left(\frac{r}{r_{H}}\right)^{2}+\frac{c^{2} \chi}{2 \alpha_{\Lambda}}\left(\frac{\Lambda_{\mathrm{DE}}}{8 \pi \rho_{H}}\right)^{\alpha_{\Lambda}}
$$

in Region I, while in Region II,

$$
\begin{aligned}
\mathfrak{V}(r)= & \Phi(0)+\frac{1}{2} v_{H}^{2}+v_{H}^{2} \log \left[\frac{r}{r_{H}}\right] \\
& -\frac{\left(1+\alpha_{\Lambda}\right)^{2}}{2\left(1+3 \alpha_{\Lambda}\right)}\left(1-\frac{r_{H}}{r}\right)\left(c^{2} \chi \Sigma\left(\alpha_{\Lambda}\right) u_{H}^{\frac{2 \alpha_{\Lambda}}{\left(1+\alpha_{\Lambda}\right)}}-6 v_{H}^{2}\right) \\
& -\frac{\left(1+\alpha_{\Lambda}\right)^{2}}{\left(1+3 \alpha_{\Lambda}\right)} \frac{c^{2} \chi}{4 \alpha_{\Lambda}}\left\{\frac{r_{H}^{2}}{\chi \lambda_{\mathrm{DE}}^{2}}\left(\frac{8 \pi \rho_{\mathrm{asymp}}\left(r_{H}\right)}{\Lambda_{\mathrm{DE}}}-\alpha_{\Lambda} \frac{8 \pi \rho_{\mathrm{II}}^{1}\left(r_{H}\right)}{\Lambda_{\mathrm{DE}}}\right)\right\},
\end{aligned}
$$


where we have used $\rho_{\mathrm{II}}^{1} / \rho_{\text {asymp }}<<1$ and Eq. (16). The effective potential thus increases only logarithmically as $r$ increases. This is expected, and is consistent with using the rotational velocity curves in constructing $f(r)$.

The $r^{1.22}$ increase in $\Phi(r)$, if unchecked, would mean that at some point the weak gravity approximation used here would be violated. Indeed, it is doubtful if the resultant spacetime will be gravitationally stable, and it would certainly be inconsistent with WMAP measurements, which indicate that the universe is flat. We found that Region III the density $\rho \rightarrow 0$ exponentially fast, however, and thus $\Phi \rightarrow 0$ exponentially fast as well when $r>r_{\mathrm{II}}$. This provides a cutoff to $\Phi(r)$, and prevents the gravitational potential from becoming infinite at large $r$. The growth in $\Phi$ ends at $r=r_{\text {II }}$, and as we find that $\Phi\left(r_{\mathrm{II}}\right)-\Phi(0) \approx 0.02 c^{2}$, the weak gravity approximation is valid for Regions I and II.

\subsection{Matter density measurements under the extended GEOM}

That the motion of stars in the galaxy is determined by $\mathfrak{V}(\mathbf{x})$ and not by $\Phi(\mathbf{x})$ has far reaching implications. The local density of matter in the universe is determine through observations on the influence that this density has on the motion of test particles. These test particles can be divided into two classes: massive particles, such as the motion of stars in galaxies, and massless particles, such as the motion of photons of various frequencies.

The extended GEOM affects only the motion of massive test particles. As noted above, the motion of such particles is determined by the effective potential, $\mathfrak{V}$. As $\mathfrak{V}$ is in turn determined by $\rho_{\mathrm{II}}^{1}$, and not the background density, $\rho_{\text {asymp }}$, observations of the rotational velocity curves of a galaxy using the motion of the stars in the galaxy will at best be able to determine the perturbation on the background density, $\rho_{\mathrm{II}}^{1}$, and not $\rho_{\text {asymp }}$ itself. Since $\rho_{\text {asymp }}(r)>>\rho_{\text {II }}^{1}(r)$ when $r>>r_{H}$, the majority of the mass in the universe cannot be seen with these methods. In particular, the motion of stars in galaxies can only be used to estimate $\rho(r)-\rho_{\text {asymp }}$; the matter in $\rho_{\text {asymp }}$ is present, but cannot be determined in this way.

The extended GEOM does not affect the motion of massless particles, however. Thus, the trajectory of photons still follow the GEOM, and these equations are determined by the local metric, $g_{\mu \nu}$. In the nonrelativistic and weak gravity limits, $g_{\mu \nu}$ is determined in turned by the potential, $\Phi(\mathbf{x})$. Consequently, measurements of the local density of matter using photons — such as through gravitational lensing-results in determining the total density, $\rho$, and not just the small fraction of it in $\rho_{\mathrm{II}}^{1}$ as is possible using massive particles.

\section{Concluding remarks}

Given how sensitive our expression for $\sigma_{8}$ is dependent on $\alpha_{\Lambda}$, that our predicted values of $\sigma_{8}$ is within experimental error of its measured value by WMAP is a compelling result. This is especially true as the data used in calculating $\sigma_{8}$ was taken by four different groups over a period of 30 years, and for purposes that have no connection whatsoever with our analysis. In the absence of a direct experimental measurement 
of $\alpha_{\Lambda}$, this agreement between the calculated and measured values of $\sigma_{8}$ provides a persuasive argument for the validity of our extension of the GEOM.

In [1], we performed a gedanken experiment based on the measurement of anomalous accelerations in a dilute gas due to the passage of a sound wave with wavenumber $k=1 \mathrm{~cm}^{-1}$, and an amplitude that is $10 \%$ that of an ambient gas that has a density of $10^{-18} \mathrm{~g} / \mathrm{cm}^{3}$. We were able to establish a rough lower bound on $\alpha_{\Lambda}$ that for $\Lambda_{\mathrm{DE}}=7.21 \times 10^{-30} \mathrm{~g} / \mathrm{cm}^{3}$ is 1.56. This is equal to the value of $\alpha_{\Lambda}$ found in Sect. 3 . While this gedanken experiment was a crude estimate of the effects of the extended GEOM and while it is an open question as to whether such an experiment would be feasible, the fact that the value of $\alpha_{\Lambda}$ found is so close to this lower bound raises the possibility that direct detection and measurement of $\alpha_{\Lambda}$ through terrestrial experiments may be possible in the near future.

Open Access This article is distributed under the terms of the Creative Commons Attribution Noncommercial License which permits any noncommercial use, distribution, and reproduction in any medium, provided the original author(s) and source are credited.

\section{Appendix A: Fitting to the Rubin et al. data set}

In [11], measurements of the rotational velocity as a function of radius for $60 \mathrm{Sa}, \mathrm{Sb}$ and Sc spiral galaxies are given. These measurements allows us to fit the data given to a model of the rotational velocity curve. Instead of fitting the data directly to the pseudo-isothermal velocity curve, $v^{\mathrm{p} \text {-iso }}(r)$, as is done in [5], it is more convenient to fit it to $\left(v^{\mathrm{p}-\text { iso }}(r)\right)^{2}$. Moreover, since what is needed is the asymptotic rotational velocity instead of the density parameter for the pseudo-isothermal profile, our fit to $\left(v^{\mathrm{p} \text {-iso }}(r)\right)^{2}=v_{H}^{2} c(r)$ uses $v_{H}$ and $R_{C}$ as the two parameters. Here,

$$
c(r)=1-\frac{R_{C}}{r} \arctan \left(\frac{r}{R_{C}}\right) .
$$

The variance of the fit is then

$$
\sigma_{(v \mathrm{p} \text {-iso })^{2}}^{2} \equiv \frac{1}{N-2} \sum_{n=1}^{N}\left[\left(v_{n}^{\mathrm{p} \text {-iso }}\right)^{2}-v_{H}^{2} c\left(r_{n}\right)\right]^{2},
$$

where $\left\{\left(v_{n}^{\mathrm{p}-\text { iso }}, r_{n}\right)\right\}$ is the set $N$ of rotational velocity verses radius measurements for a galaxy. A least squares fit gives

$$
v_{H}^{2}=\frac{\left\langle\left(v_{n}^{\mathrm{p}-\text { iso }}\right)^{2} c\left(r_{n}\right)\right\rangle}{\left\langle c\left(r_{n}\right)^{2}\right\rangle},
$$

where $\langle\cdots\rangle$ denotes an average over the data points. While an equation for $R_{C}$ can also be given through minimization of Eq. (39), the resultant equation for $R_{C}$ is given implicitly. We find it more useful to substitute Eq. (40) into Eq. (39), and then use iteration to find the $R_{C}$ that minimizes $\sigma_{\left(v \mathrm{p}^{\mathrm{p}} \text { iso }\right)^{2}}^{2}$. Using this value for $R_{C}, v_{H}^{2}$ is then given by Eq. (40). 
The standard error, $\sigma_{v_{H}}$, in $v_{H}$, is

$$
\begin{aligned}
\sigma_{v_{H}}= & \frac{1}{2}\left(\frac{\left.\sigma^{2} v_{n}^{\text {p-iso }}\right)^{2}}{N v_{H}^{2}\left\langle c\left(r_{n}\right)^{2}\right\rangle}\right. \\
+ & {\left.\left[1+\frac{1}{v_{H}^{2}\left\langle c\left(r_{n}\right)^{2}\right\rangle}\left\langle\frac{v_{n}^{\mathrm{p}-\text { iso }} r_{n}^{2}}{R_{C}^{2}+r_{n}^{2}}\right\rangle-\frac{2}{\left\langle c\left(r_{n}\right)^{2}\right\rangle}\left\langle\frac{c\left(r_{n}\right) r_{n}^{2}}{R_{c}^{2}+r_{n}^{2}}\right\rangle\right]^{2} \frac{\sigma_{R_{C}}^{2}}{R_{c}^{2}}\right)^{\frac{1}{2}}, }
\end{aligned}
$$

while the standard error, $\sigma_{R_{C}}$, in $R_{C}$ is

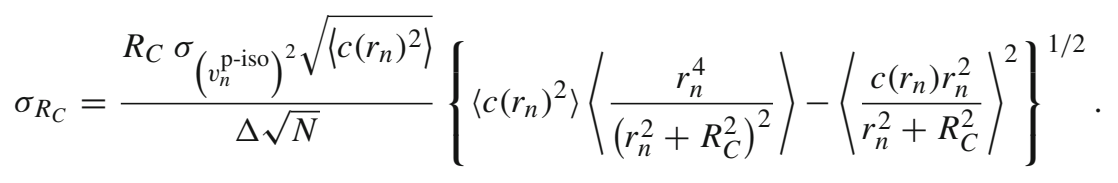

Here,

$$
\begin{aligned}
\Delta \equiv & 2\left\langle\left(v_{n}^{\mathrm{p}-\text { iso }}\right)^{2} c\left(r_{n}\right)\right\rangle\left\langle\frac{c\left(r_{n}\right) r_{n}^{4}}{\left(r_{n}^{2}+R_{C}^{2}\right)^{2}}\right\rangle-2\left\langle c\left(r_{n}\right)^{2}\right\rangle\left\langle\frac{\left(v_{n}^{\mathrm{p}-\text { iso }}\right)^{2} r_{n}^{4}}{\left(r_{n}^{2}+R_{C}^{2}\right)^{2}}\right\rangle \\
& +\left\langle\frac{c\left(r_{n}\right) r_{n}^{2}}{r_{n}^{2}+R_{C}^{2}}\right\rangle\left\langle\frac{\left(v_{n}^{\mathrm{p}-\text { iso }}\right)^{2} r_{n}^{2}}{r_{n}^{2}+R_{C}^{2}}\right\rangle-\left\langle\left(v_{n}^{\mathrm{p}-\text { iso }}\right)^{2} c\left(r_{n}\right)\right\rangle\left\langle\frac{r_{n}^{4}}{\left(r_{n}^{2}+R_{C}^{2}\right)^{2}}\right\rangle .
\end{aligned}
$$

The results of our fits of the Rubin et al. data are tabulated in Table 2. The base data from [11] used a Hubble constant of $50 \mathrm{~km} \mathrm{~s}^{-1} \mathrm{Mpc}^{-1}$, and the results given in the table are for this value of the constant. Of the 60 galaxies from [11], NGC 6314 and IC 724 could not be fitted to a nonzero $R_{C}$, while the fit for NGC 2608 resulted in a $R_{C}$ that is less than $0.01 \mathrm{kpc}$.

\section{Appendix B: Data sets}

For the de Blok et al. data set, the galaxy F568-3 was analyzed twice; we use the analysis of F568-3 given by the authors in [5]. In de Blok and Bosma [6], two of the galaxies, F563-1 and U5750, also appeared in [5]; we used the values from [5] for these galaxies in our analysis. Finally, the radius for DDO185 from [5] was not determined, and we could not include this data point in our analysis. Thus, out of 56 galaxies in this data set, 53 were used.

For the Rubin et al. data set, we could not find a nonzero radius for two galaxies, and one galaxy had a radius less than $0.01 \mathrm{kpc}$. As this radius was smaller than the resolution of their observations, this data point was not included. A total of 57 galaxies were thus used from [11]. 
Table 2 Fitted values of $R_{C}(\mathrm{kpc})$ and $v_{H}(\mathrm{~km} / \mathrm{s})$, and their errors for the Rubin et al. data set

\begin{tabular}{|c|c|c|c|c|c|c|c|c|c|}
\hline Galaxy & $R_{C}$ & $\Delta R_{C}$ & $v_{H}$ & $\Delta v_{H}$ & Galaxy & $R_{C}$ & $\Delta R_{C}$ & $v_{H}$ & $\Delta v_{H}$ \\
\hline NGC 1024 & 0.27 & 0.14 & 229.42 & 9.77 & NGC 4800 & 0.18 & 0.06 & 171.56 & 3.42 \\
\hline NGC 1357 & 0.52 & 0.14 & 268.19 & 16.27 & NGC 7083 & 0.89 & 0.14 & 226.51 & 2.27 \\
\hline NGC 2639 & 1.02 & 0.34 & 337.69 & 31.31 & NGC 7171 & 2.25 & 0.36 & 251.35 & 6.47 \\
\hline NGC 2775 & 0.40 & 0.17 & 298.98 & 8.90 & NGC 7217 & 0.19 & 0.10 & 275.21 & 6.56 \\
\hline NGC 2844 & 0.41 & 0.09 & 167.50 & 18.93 & NGC 7537 & 0.80 & 0.10 & 150.06 & 2.35 \\
\hline NGC 3281 & 0.44 & 0.05 & 211.32 & 26.51 & NGC 7606 & 1.40 & 0.30 & 279.29 & 4.11 \\
\hline NGC 3593 & 0.16 & 0.08 & 115.28 & 14.01 & UGC 11810 & 1.54 & 0.38 & 193.28 & 3.85 \\
\hline NGC 3898 & 0.53 & 0.06 & 254.76 & 28.73 & UGC 12810 & 3.22 & 0.35 & 245.73 & 1.47 \\
\hline NGC 4378 & 0.13 & 0.06 & 307.61 & 26.60 & $\mathrm{NGC}^{-}$ & 2.49 & 0.58 & 188.78 & 4.86 \\
\hline NGC 4419 & 0.63 & 0.03 & 211.55 & 2.33 & NGC 753 & 0.31 & 0.11 & 208.50 & 3.57 \\
\hline NGC 4594 & 1.65 & 0.30 & 397.24 & 10.15 & NGC 801 & 0.79 & 0.16 & 227.64 & 4.06 \\
\hline NGC 4698 & 1.85 & 0.47 & 284.96 & 6.34 & NGC 1 & 1.24 & 0.09 & 150.62 & 1.26 \\
\hline NGC 4845 & 0.11 & 0.07 & 187.54 & 0.07 & NGC 1087 & 0.54 & 0.10 & 131.91 & 2.54 \\
\hline UGC 10205 & 2.19 & 0.27 & 272.34 & 4.07 & NGC 1421 & 0.54 & 0.13 & 176.42 & 3.94 \\
\hline NGC 1085 & 0.29 & 0.05 & 307.02 & 2.11 & NGC 2715 & 1.10 & 0.22 & 151.47 & 2.93 \\
\hline NGC 1325 & 1.80 & 0.28 & & 2.67 & & 1.10 & 0.16 & & 2.36 \\
\hline NGC 1353 & 0.36 & 0.18 & 218.48 & 8.30 & NGC 2998 & 1.08 & 0.22 & 213.85 & 3.22 \\
\hline NGC 1417 & 0.40 & 0.05 & 278.87 & 2.36 & NGC 3495 & 3.11 & 0.46 & 206.75 & 3.22 \\
\hline NGC 1515 & 0.06 & 0.10 & 178.35 & 10.03 & NGC 3672 & 1.74 & 0.24 & 208.11 & 4.03 \\
\hline NGC 1620 & 1.73 & 0.25 & & 3.14 & $\mathrm{NGC}$ & 0.79 & 0.13 & & 2.65 \\
\hline NGC 2590 & 1.30 & 0.54 & 255.24 & 5.33 & NGC 4321 & 0.79 & 0.35 & 208.24 & 5.42 \\
\hline NGC 2708 & 1.91 & 0.68 & 269.92 & 9.45 & NGC 4605 & 0.97 & 0.32 & 112.62 & 3.42 \\
\hline NGC 2815 & 1.91 & 0.68 & 269.92 & 9.45 & NGC 4682 & 1.17 & 0.23 & 181.17 & 2.97 \\
\hline NGC 3054 & 2.41 & 0.56 & 259.10 & 8.30 & NGC 7541 & 0.21 & 0.16 & 195.04 & 5.94 \\
\hline NGC 3067 & 0.76 & 0.06 & 156.80 & 1.22 & NGC 7664 & 0.65 & 0.14 & 196.05 & 3.07 \\
\hline NGC 3145 & 0.15 & 0.07 & 257.00 & 4.84 & IC 467 & 1.64 & 0.33 & 152.42 & 3.26 \\
\hline NGC 3200 & 0.42 & 0.09 & 266.07 & 5.43 & UGC 2885 & 0.06 & 0.10 & 266.22 & 5.88 \\
\hline NGC 3223 & 1.35 & 0.23 & 275.29 & 5.51 & UGC 3691 & 3.04 & 0.33 & 229.42 & 1.31 \\
\hline NGC 4448 & 0.59 & 0.11 & 207.02 & 1.98 & & & & & \\
\hline
\end{tabular}

For the CF and Mathewson et al. data sets, the vast majority of the data were based on single observations. While we may have greater leeway in removing outliers from this data set, even here we were circumspect. First, 75 galaxies in [12] were observed multiple times. Of these, the galaxies UGC 7234 and UGC 10096 had listed an asymptotic velocity for one of the observations that was opposite from the measured asymptotic velocity for the others. We assumed that this was a typographical error, and the sign of the anomalous rotational velocity is reversed. Second, five galaxies in the $\mathrm{CF}$ and Mathewson et al. data sets had a $r_{H}=0$, one galaxy had a radius that was 11 -sigma out from the mean, and three galaxies had a $v_{H}$ that exceeded $8,000 \mathrm{~km} / \mathrm{s}$. These are likely indications that the data was not sufficiently accurate to allow for a 
Table 3 Listed are the galaxies removed from the data sets used in our analysis along with the reason for their removal

\begin{tabular}{|c|c|c|}
\hline Data set & Data removed & Reason \\
\hline de Blok et al. & DDO185 & $r_{H}=\infty$ \\
\hline \multirow[t]{3}{*}{ Rubin et al. } & NGC 6314 & $r_{H}=0$ \\
\hline & IC 724 & $r_{H}=0$ \\
\hline & NGC 2608 & $r_{H}<0.01$ \\
\hline \multirow[t]{2}{*}{$\mathrm{CF}$} & UGC 6534 & $v_{H}$ is $35 \sigma$ out \\
\hline & UGC 12543 & $v_{H}$ is $11 \sigma$ out \\
\hline \multirow[t]{21}{*}{ Mathewson et al. } & ESO 140-G28 & $v_{H}>8,000 \mathrm{~km} / \mathrm{s}$ \\
\hline & ESO 481-G30 & $v_{H}>24,000 \mathrm{~km} / \mathrm{s}$ \\
\hline & ESO 443-G42 & $v_{H}>94,000 \mathrm{~km} / \mathrm{s}$ \\
\hline & ESO 108-G19 & $r_{H}=0$ \\
\hline & ESO 141-G34 & $r_{H}=0$ \\
\hline & ESO 21-G5 & $r_{H}$ is $6 \sigma$ out \\
\hline & ESO 548-G21 & $r_{H}$ is $7 \sigma$ out \\
\hline & NGC 7591 & $r_{H}=0$ \\
\hline & ESO 243-G34 & $v_{H}$ is $5 \sigma$ out \\
\hline & ESO 317-G41 & $r_{H}=0$ \\
\hline & ESO 358-G9 & $v_{H}$ is $6 \sigma$ out \\
\hline & ESO 435-G25 & $v_{H}$ is $5 \sigma$ out \\
\hline & ESO 467-G12 & $r_{H}=0$ \\
\hline & ESO 554-G28 & $v_{H}$ is $6 \sigma$ out \\
\hline & ESO 60-G24 & $v_{H}$ is $10 \sigma$ out \\
\hline & ESO 359-G6 & $r_{H}$ is $11 \sigma$ out \\
\hline & ESO 481-G21 & $r_{H}$ is $6 \sigma$ out \\
\hline & UGCA 394 & $r_{H}$ is $7 \sigma$ out \\
\hline & ESO 298-G15 & $r_{H}$ is $7 \sigma$ out \\
\hline & ESO 545-G3 & $r_{H}$ is $7 \sigma$ out \\
\hline & ESO 404-G18 & $r_{H}$ is $9 \sigma$ out \\
\hline
\end{tabular}

fit of the velocity curve, and these galaxies were removed from the data sets. Finally, given that there are only 1,393 galaxies combined in the data sets, if a galaxy had a $v_{H}$ or a $r_{H}$ that was five-sigma or more out from the mean, it was removed. In the end, 348 galaxies were used in the CF data set, and 935 galaxies were used in the Mathewson et al. data set. A summary of the data points not used in our analysis is given in Table 3.

\section{References}

1. Speliotopoulos, A.D.: Gen. Relativ. Gravit. 42, 1537 (2010)

2. Riess, A.G., Filippenko, A.V., Challis, P., Clocchiatti, A., Diercks, A., Garnavich, P.M., Gilliland, R.L., Hogan, C.J., Jha, S., Kirshner, R.P., Leibundgut, B., Phillips, M.M., Riess, D., Schmidt, B.P., Schommer, R.A., Smith, R.C., Spyromilio, J., Stubbs, C., Suntzeff, N.B., Tonry, J.: Astron. J 116, 1009 (1998) 
3. Perlmutter, S., Aldering, G., Goldhaber, G., Knop, R.A., Nugent, P., Castro, P.G., Deustua, S., Fabbro, S., Goobar, A., Groom, D.E., Hook, I.M., Kim, A.G., Kim, M.Y., Lee, J.C., Nunes, N.J., Pain, R., Pennypacker, C.R., Quimby, R., Lidman, C., Ellis, R., Irwin, M., McMahon, R.G., RuizLapuente, P., Walton, N., Schaefer, B., Boyle, B.J., Filippenko, A.V., Matheson, P., Fruchter, A.S., Panagia, N., Newberg, H.J.M., Couch, W.J.: Astrophys. J. Suppl. 517, 565 (1999)

4. Spergel, D.N., Bean, R., Doré, O., Nolta, M.R., Bennett, C.L., Dunkley, J., Hinshaw, G., Jarosik, N., Komatsu, E., Page, L., Peiris, H.V., Verde, L., Halpern, M., Hill, R.S., Kogut, A., Limon, M., Meyer, S.S., Odegard, N., Tucker, G.S., Weiland, J.L., Wollack, E., Wright, E.L.: Astrophys. J. Suppl. 170, 277 (2007)

5. de Blok, W.J.G., McGaugh, S.S., Bosma, A., Rubin, V.C.: Astrophys. J 552, L23 (2001)

6. de Blok, W.J.G., Bosma, A.: Astro. Astrophys. 385, 816 (2002)

7. McGaugh, S.S., Rubin, V.C., de Blok, W.J.G.: Astron. J 122, 2381 (2001)

8. Rubin, V.C., Ford, W.K. Jr., Thonnard, N.: Astrophys. J 238, 471 (1980)

9. Rubin, V.C., Ford, W.K. Jr., Thonnard, N., Burstein, D.: Astrophys. J 261, 439 (1982)

10. Burstein, D., Rubin, V.C., Thonnard, N., Ford, W.K. Jr.: Astrophys. J. Part 1 253, 70 (1982)

11. Rubin, V.C., Burstein, D., Ford, W.K. Jr., Thonnard, N.: Astrophys. J 289, 81 (1985)

12. Courteau, S.: Astron. J 114, 2402 (1997)

13. Mathewson, D.S., Ford, V.L., Buchhorn, M.: Astrophys. J. Suppl. 82, 413 (1992)

14. Peebles, P.J.E.: The Large-Scale Structure of the Universe, Chapter 2,3. Princeton University Press, Princeton (1980)

15. Fillmore, J.A., Goldreich, P.: Astrophys. J 281, 1 (1984)

16. Hoffman, Y., Shaham, J.: Astrophys. J 297, 16 (1985)

17. Hoffman, Y.: Astrophys. J 328, 489 (1988)

18. Persic, M., Salucci, P.: Astron. J. Suppl. 99, 501 (1995)

19. Persic, M., Salucci, P., Stel, F.: Mon. Not. R. Astron. Soc. 281, 21 (1996)

20. Bender, C.M., Orszag, S.A.: Advanced Mathematical Methods for Scientists and Engineers. McGraw-Hill Book Company, New York (1978)

21. Davis, T., Lineweaver, C.H.: Publ. Astron. Soc. Aust. 21, 97 (2004)

22. Kolb, E.W., Turner, M.: The Early Universe, Chap. 9. Addison-Wesley, New York (1990)

23. McGaugh, S.S.: Astron. J 632, 859 (2005) 